\title{
An unconventional exit for Brucella
}

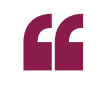

the $\mathrm{rBCV}$

matures into an

autophagosome-

like vacuole

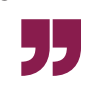

During the early part of its intracellular life cycle, Brucella abortus resides in a membrane-bound compartment known as the Brucella-containing vacuole (BCV). The BCV traffics through the endocytic pathway to the endoplasmic reticulum (ER), where it is known as the replicative $B C V(r B C V)$, in which the bacterium proliferates. Celli and colleagues now delineate what occurs during the post-replicative stages of infection and show that the rBCV matures into a compartment with autophagic features.

First, the authors detected a compartment that was highly prevalent during late stages of the life cycle ( 72 hours post-infection) and that contained live bacteria. This compartment was phenotypically distinct from the $\mathrm{rBCV}$, being positive for lysosome-associated membrane protein 1 (LAMP1) and negative for calreticulin. Furthermore, unlike the rBCV, this compartment was delimited by a double membrane, similarly to autophagosomes. These observations

\section{indicate that, after B. abortus} replication, the rBCV matures into an autophagosome-like vacuole, which the authors term autophagic BCV (aBCV).

Canonical autophagosome formation involves four steps - initiation, nucleation, elongation and recycling - that depend on distinct autophagy proteins. Depletion of the initiation protein ULK1 (UNC51-like kinase 1) and/or the nucleation protein BECLIN1 by small interfering RNAs (siRNAs) significantly decreased aBCV formation. By contrast, no impairment was observed following loss of the elongation proteins autophagy-related 5 (ATG5), ATG7 and light chain 3 (LC3), either using siRNAs or in mutant mice. Thus, aBCV formation is independent of the elongation proteins and may be an example of non-canonical autophagosome formation. Notably, depletion of ULK1 and/or BECLIN1, and of ATG5, ATG7 and LC3 did not affect the earlier step of maturation into the rBCV.

BECLIN1, together with phosphoinositide 3-kinase (PI3K), forms an ER-localized complex that initiates the biogenesis of autophagic membranes or a complex that localizes to endocytic compartments and regulates autophagosome maturation. Depletion of ATG14L (also known as BARKOR; which localizes the complex to the ER) but not of UVRAG (which localizes the complex to the endocytic compartment) decreased $\mathrm{aBCV}$ formation. This indicates that aBCV formation is initiated by the ER-localized BECLIN1 complex.

So what is the role of aBCVs? Because they occur late in the B. abortus life cycle, the authors postulated that aBCVs contribute to bacterial egress and infection of neighbouring cells, thereby completing the bacterial life cycle. Indeed, they observed that infected cells generate infection foci (as monitored by the infection of neighbouring cells) by 72 hours post-infection, and these foci were associated with aBCVs. Moreover, depletion of BECLIN1, ULK1 or both, but not of the elongation proteins, significantly decreased the formation of infection foci.

These findings reveal that $B$. abortus hijacks components of the autophagy machinery to generate vacuoles that facilitate its egress from host cells and its infection of neighbouring cells, completing the infectious cycle. Further work is needed to elucidate the signals that initiate $\mathrm{aBCV}$ formation and the molecular mechanisms of bacterial exit. Rachel David

ORIGINAL RESEARCH PAPER Starr, T. et al. Selective subversion of autophagy complexes facilitates completion of the Brucella intracellular cycle. Cell Host Microbe 11, 33-45 (2012) 